\title{
Condutância vascular aumentada em indivíduos fisicamente ativos filhos de hipertensos
}

\author{
Vascular conductance increased in physically active children of \\ hypertensive individuals
}

Leonardo Barbosa de Almeida', Isabelle Magalhães Guedes Freitas', Livia Victorino de Souza', Pedro Augusto de Carvalho Mira', Daniel Godoy Martinez', Mateus Camaroti Laterza’

\begin{abstract}
Resumo
Objetivou-se testar a hipótese de que a prática regular de exercício físico melhora a função vascular de filhos de hipertensos em repouso. Foram avaliados 13 indivíduos fisicamente ativos filhos de hipertensos (FHA) e 22 indivíduos sedentários filhos de hipertensos (FHS), pareados por idade $(22,5 \pm 2,9$ vs. $23,8 \pm 2,7$ anos, $\mathrm{p}=0,18)$ e $\operatorname{IMC}\left(23,8 \pm 1,9\right.$ vs. $23,0 \pm 3,0 \mathrm{~kg} / \mathrm{m}^{2}$, $\mathrm{p}=0,45)$. Foram registrados, simultaneamente, a frequência cardíaca, a pressão arterial, minuto a minuto (método oscilométrico - DIXTAL $2022^{\circledR}$ ) e o fluxo sanguíneo do antebraço (pletismografia de oclusão venosa - Hokanson ${ }^{\circledR}$ ), continuamente, durante 3 minutos em repouso. A função vascular foi avaliada por meio da condutância vascular do antebraço, calculada pela divisão do fluxo sanguíneo do antebraço pela pressão arterial média e multiplicada por 100. Foi adotado significância de $\mathrm{p} \leq 0,05$. Como resultados, em condições basais, os grupos FHA e FHS foram semelhantes para pressão arterial sistólica $(124 \pm 9$ vs. $121 \pm 11 \mathrm{mmHg}, \mathrm{p}=0,42)$, diastólica $(64 \pm 5$ vs. $64 \pm 5 \mathrm{mmHg}, \mathrm{p}=0,94)$, média $(84 \pm 6$ vs. $83 \pm 7$ $\mathrm{mmHg}, \mathrm{p}=0,71)$ e fluxo sanguíneo do antebraço $(3,6 \pm 1,1$ vs. $2,9 \pm 0,9 \mathrm{ml} / \mathrm{min} / 100 \mathrm{ml}, \mathrm{p}=0,06)$. Mas, o grupo FHA apresentou menor valor de frequência cardíaca de repouso (61 \pm 7 vs. $70 \pm 8$ bpm, $\mathrm{p}<0,01)$ e maior condutância vascular do antebraço $(4,3 \pm 1,3$ vs. $3,4 \pm 1,0$ unidades, $\mathrm{p}=0,05)$. Conclui-se que indivíduos fisicamente ativos filhos de hipertensos apresentam melhor função vascular em situação de repouso.
\end{abstract}

\section{Palavras-chave}

Hipertensão; Hereditariedade; Capacitância vascular.

\begin{abstract}
The aim of this study was to test the hypothesis that regular physical exercise practice improves vascular function at rest of bypertensive parents' offspring. We evaluated 13 physically active offspring of bypertensive (FHA) parents and 22 sedentary offspring of bypertensive (FHS), matched by age $(22.5 \pm 2.9$ vs. $23.8 \pm 2.7$ years, $p=0.18)$ and BMI $\left(23.8 \pm 1.9\right.$ vs. $\left.23.0 \pm 3.0 \mathrm{~kg} / \mathrm{m}^{2}, p=0.45\right)$. Heart rate, minute by minute, blood pressure (oscillometric method - DIXTAL 2022 ${ }^{\circledR}$ ), and forearm blood flow (venous occlusion plethysmography-Hokanson ${ }^{\circledR}$ ) were continuously recorded for 3 minutes at rest. Vascular function was assessed by forearm vascular conductance, which was calculated by the division of forearm blood flow by mean arterial pressure, multiplied by 100. A p-value $<0.05$ was considered statistically significant. At rest condition, the groups were similar for systolic (124 \pm 9 vs. $121 \pm 11 \mathrm{mmHg}, p=0.42)$, diastolic ( $64 \pm 5$ vs. $64 \pm 5 \mathrm{mmHg}, p=0.94)$, mean blood pressure (84 \pm 6 vs. $83 \pm 7 \mathrm{mmHg}, p=0.71$ ) and forearm blood flow (3.6 \pm 1.1 vs. $2.9 \pm 0.9 \mathrm{ml} / \mathrm{min} / 100 \mathrm{ml}, p=0.06)$. On the other hand, FHA group showed lower resting heart rate $(61 \pm 7$ vs. $70 \pm 8 \mathrm{bpm}$, $p^{<0,01)}$ and higher forearm vascular conductance $(4.3 \pm 1.3 \mathrm{vs}$. $3.4 \pm 10$ units, $p=0.05$ ) than FHS group. We concluded that physically active offspring of bypertensive parents presents improved vascular function at rest in comparison with sedentary ones.
\end{abstract}

\section{Keywords}

Hypertension; Heredity; Vascular Capacitance.

\section{Introdução}

O histórico familiar positivo para hipertensão arterial tem sido considerado um dos principais fatores da gênese hipertensiva ${ }^{1-4}$. Diversos estudos ${ }^{5-8}$ têm de-

1 Unidade de Investigação Cardiovascular e Fisiologia do Exercício do Hospital Universitário e da Faculdade de Educação Física e Desportos da Universidade Federal de Juiz de Fora, Juiz de Fora, MG, Brasil. monstrado que mesmo dentro da faixa de normalidade para classificação diagnóstica, pessoas normotensas com pai e/ou mãe com hipertensão possuem valores significativamente maiores de pressão arterial quando comparadas às pessoas também normotensas, mas com o pai e mãe normotensos. Associado ao maior valor pressórico, Wang et al. ${ }^{1}$ investigaram o 
impacto da hipertensão arterial dos pais nas alterações nos níveis pressóricos e no risco de desenvolvimento de hipertensão em 1160 homens normotensos durante 54 anos de seguimento. Nesse estudo, o risco relativo de desenvolvimento de hipertensão foi de 1,5, 1,8 e 2,4 para os indivíduos que possuíam, respectivamente, somente a mãe, somente o pai e ambos os pais hipertensos, comparativamente aos indivíduos cujos pais eram normotensos.

Alterações nos mecanismos de controle hemodinâmico parecem, pelo menos em parte, estarem envolvidos na relação hereditariedade e desenvolvimento de hipertensão arterial. De fato, diversos estudos têm demonstrado que tanto a estrutura $^{8-10}$ como a função vascular ${ }^{9,11}$ já estão prejudicadas nos indivíduos normotensos filhos de hipertensos. Por exemplo, Ciolac et al. ${ }^{8}$ avaliaram mulheres jovens filhas de apenas um dos pais hipertensos e filhas de ambos os pais hipertensos e observaram que a velocidade de onda de pulso, marcador de rigidez arterial, estava aumentada em ambos os grupos quando comparadas às mulheres sem histórico para a doença. E mais, não só a estrutura vascular dos filhos de hipertensos encontra-se prejudicada, mas também sua função. Nesse cenário, Evrengul et al. ${ }^{9}$ observaram diminuição da vasodilatação dependente do endotélio, por meio da técnica de hiperemia reativa, em filhos de hipertensos comparados a filhos de normotensos.

Por outro lado, sabe-se que a prática regular de exercício físico promove importantes adaptações no sistema vascular. Estudos envolvendo seres humanos saudáveis e em condições patológicas demonstraram que o treinamento físico é capaz de promover remodelamento vascular, aumentando a elasticidade $\operatorname{arterial}^{12,13}$ e a vasodilatação endotélio-dependente ${ }^{15-16}$, tendo papel preponderante na redução dos níveis pressóricos.

Assim, é possível pensarmos que a prática regular de exercício físico pode reduzir a influência negativa que o histórico familiar positivo para hipertensão exerce sobre os vasos arteriais. Dessa forma, o objetivo do presente estudo foi testar a hipótese de que indivíduos fisicamente ativos filhos de hipertensos apresentam melhor função vascular quando comparados a indivíduos sedentários filhos de hipertensos.

\section{Métodos}

\section{Casuística}

O cálculo da amostra foi baseado em estudo previamente publicado ${ }^{14}$, considerando poder de $90 \%$ e erro $\alpha$ de $5 \%$. Por esse cálculo, deveriam ser recrutados, no mínimo, 12 voluntários para cada grupo. Portanto, para esse estudo transversal, foram selecionados 35 indivíduos com histórico familiar positivo para hipertensão arterial sistêmica. Os indivíduos recrutados deveriam apresentar as seguintes características: ter um ou ambos os pais hipertensos, ser normotenso, idade entre 18 e 40 anos, não ser tabagista e/ou etilista e não apresentar diagnóstico médico de qualquer doença cardiorrespiratória e/ou metabólica. Os voluntários foram divididos em dois grupos de acordo com a prática regular de exercício físico: 13 indivíduos fisicamente ativos (Grupo FHA) e 22 indivíduos sedentários (Grupo FHS).

Todos os voluntários foram submetidos à entrevista, avaliação da prática de exercício físico, avaliação da pressão arterial, frequência cardíaca e fluxo sanguíneo do antebraço.

Todas as variáveis dos voluntários envolvidos nessa pesquisa foram coletadas do banco de dados formado por duas pesquisas previamente aprovadas pelo Comitê de Ética em Pesquisa com Seres Humanos do Hospital Universitário da Uni- 
versidade Federal de Juiz de Fora (HU/UFJF), Juiz de Fora, MG, Brasil (0113/2010 e 0119/2010), de acordo com a resolução 196/96 do Conselho Nacional de Saúde. Assim, todos os voluntários concordaram e assinaram os respectivos termos de consentimento livre e esclarecido.

\section{Procedimento experimental}

A pesquisa foi realizada no laboratório da Unidade de Investigação Cardiovascular e Fisiologia do Exercício do HU/UFJF e conduzida sempre no mesmo período do dia (entre 13:00 e 18:00 horas). Para a véspera e no dia das avaliações, cada voluntário recebeu orientações para evitar consumo de bebidas cafeinadas e alcoólicas, não realizar atividade física vigorosa 24 horas antes das avaliações, realizar refeições leves e ter uma noite adequada de sono (mínimo de 8 horas).

Os voluntários foram familiarizados com os pesquisadores e com o ambiente do experimento. Antes do início do protocolo experimental, todos foram avaliados e questionados para garantia de que todas as orientações haviam sido seguidas rigorosamente. Inicialmente, foi verificada a pressão arterial sistólica e diastólica pelo método auscultatório. Em seguida, os participantes foram posicionados em posição supina com a instrumentação para realização da pletismografia de oclusão venosa, da monitorização eletrocardiográfica e da medida de pressão arterial. Após o intervalo de 15 minutos de repouso, as medidas de fluxo sanguíneo, pressão arterial e frequência cardíaca foram realizadas simultaneamente durante 3 minutos.

\section{Avaliação do histórico familiar para hipertensão arterial}

Foi realizada entrevista para conhecimento dos hábitos de vida do voluntário, bem como seu estado de saúde geral e histórico familiar para doenças cardiovasculares. Nessa entrevista, foi considerado apto para pesquisa, o participante que relatava ser filho de pai e/ou mãe diagnosticado(s) com hipertensão arterial sistêmica, independentemente do estágio e do tratamento em vigência.

\section{Avaliação da prática regular de exercício físico}

O nível de atividade física dos voluntários foi avaliado por meio do questionário de Baecke ${ }^{17}$. Este é dividido em três partes: atividade física ocupacional, exercício físico nas horas de lazer e atividades físicas de lazer e locomoção, mensurados por escores específicos. O nível de atividade física nos últimos seis meses foi quantificado considerando tipo, intensidade, duração e frequência da atividade física. A partir desse questionário, foi considerada prática de exercício físico regular os casos em que o indivíduo realizasse exercícios físicos programados com frequência maior que duas vezes na semana por período maior ou igual a seis meses nos últimos 12 meses.

Além disso, o tempo gasto com exercício físico programado foi registrado individualmente.

\section{Avaliação da função vascular}

A função vascular foi avaliada pela técnica de pletismografia de oclusão venosa da marca Hokanson ${ }^{\circledR}$. Tal técnica visa medir o fluxo sanguíneo do antebraço, calcular a condutância vascular do antebraço e, assim, avaliar a função vascular. Para isso, o voluntário foi posicionado em decúbito dorsal com o braço não dominante elevado acima do nível do coração, garantindo adequada drenagem venosa. Um tubo silástico preenchido por mercúrio, conectado ao transdutor de baixa pressão e ao pletismógrafo, foi colocado ao redor do antebraço, a $5 \mathrm{~cm}$ de distância da articulação 
úmero-radial. Um manguito foi posicionado ao redor do punho e outro na parte superior do braço do voluntário. O manguito posicionado no punho foi inflado em nível de pressão supra sistólica $(200 \mathrm{mmHg})$ antes de iniciarem as medidas e mantido insuflado durante todo o procedimento. Em ciclos de 15 segundos, o manguito posicionado no braço foi inflado em nível de pressão supra venosa $(60 \mathrm{mmHg}$ ) pelo período de 7 segundos e, em seguida, foi desinsuflado rapidamente e mantido pelo mesmo tempo. Esse procedimento totalizou 4 ciclos por minuto. $\mathrm{O}$ aumento da tensão no tubo silástico refletiu o aumento de volume do antebraço e, consequentemente, o aumento no fluxo sanguíneo do antebraço, avaliado em $\mathrm{ml} / \mathrm{min} / 100 \mathrm{ml}$. O sinal da onda de fluxo sanguíneo do antebraço foi adquirido em tempo real em um computador pelo programa Non Invasive Vascular Program 3. A condutância vascular do antebraço foi calculada pelo fluxo sanguíneo do antebraço dividido pela pressão arterial média, multiplicado por 100 e reportada em unidades ${ }^{18}$.

\section{Avaliação da pressão arterial e da frequência cardíaca}

A pressão arterial não invasiva foi aferida automaticamente no membro inferior direito, com o indivíduo na posição supina, pelo método oscilométrico por meio do monitor multiparamétrico DIXTAL ${ }^{\circledR}$, modelo 2022. A frequência cardíaca foi registrada continuamente por meio de cinco eletrodos cutâneos, posicionados de acordo com a derivação padrão fornecida pelo cabo de cinco vias do mesmo monitor multiparamétrico ${ }^{18}$.

\section{Análise estatística}

Os dados serão apresentados como média \pm desvio padrão do valor médio.

A normalidade dos dados foi verificada pelo teste Kolmogorov-Smirnov. As características dos grupos investigados e os dados avaliados foram verificados pelo teste t de Student para amostras independentes. As proporções dos sexos e de número de pais hipertensos entre os grupos foram analisadas pelo teste qui-quadrado. Para esses testes, foi considerada diferença significativa $\mathrm{p} \leq 0,05$.

Além disso, foi calculado o tamanho do efeito d de Cohen. Para esse cálculo, considerou-se tamanho de efeito pequeno quando valor de $\mathrm{d}$ de Cohen foi menor que 0,2 , médio de 0,2 a 0,5 , elevado quando maior que 0,5 e menor que 1,0 e muito elevado quando foi maior que 1,0 .

\section{Resultados}

As características físicas e hemodinâmicas estão apresentadas na tabela 1. Os grupos foram semelhantes em relação ao sexo, número de pais hipertensos, idade e IMC. Como esperado, o grupo FHA apresentou valores maiores de escore de atividade física habitual. Foram semelhantes entre os grupos FHA e FHS, pressão arterial sistólica, diastólica e média. O grupo FHA apresentou frequência cardíaca significativamente menor quando comparado ao grupo FHS. O fluxo sanguíneo do antebraço foi semelhante entre os grupos FHA e FHS (Figura 1). Porém, a condutância vascular do antebraço foi significativamente maior no grupo FHA (Figura 2).

A tabela 2 apresenta os resultados obtidos para o cálculo do tamanho do efeito. Dessa forma, o tamanho do efeito foi classificado como grande tanto para o fluxo sanguíneo quanto para a condutância vascular do antebraço (Tabela 2). 
TABELA 1 - Características demográficas e hemodinâmicas da população estudada.

\begin{tabular}{lccc}
\hline & $\begin{array}{c}\text { FHA } \\
(n=13)\end{array}$ & $\begin{array}{c}\text { FHS } \\
(n=22)\end{array}$ & $p$ \\
\hline Características demográficas & & & 0,06 \\
\hline Sexo (homem/mulher) & $7 / 6$ & $24 \pm 17$ & 0,18 \\
\hline Idade (anos) & $23 \pm 3$ & $23 \pm 3$ & 0,45 \\
\hline IMC (kg/m²) & $24 \pm 2$ & $18 / 4$ & 0,39 \\
\hline No de pais hipertensos (1/2) & $12 / 1$ & $7 \pm 1$ & $<0,01$ \\
\hline NAFH (escore total) & $9 \pm 1$ & --- \\
\hline Exercício físico regular (h/sem) & $13 \pm 8$ & & \\
\hline Variáveis hemodinâmicas & & $121 \pm 11$ & 0,42 \\
\hline PAS (mmHg) & $124 \pm 9$ & $64 \pm 5$ & 0,94 \\
\hline PAD (mmHg) & $64 \pm 5$ & $83 \pm 7$ & 0,71 \\
\hline PAM (mmHg) & $84 \pm 6$ & $70 \pm 8$ & $<0,01$ \\
\hline FC (bpm) & $61 \pm 7$ & & - \\
\hline
\end{tabular}

Valores apresentados como média \pm DP. FHA = fisicamente ativos; FHS = sedentários; IMC = índice de massa corporal; NAFH = nível de atividade física habitual; PAS = pressão arterial sistólica; PAD = pressão arterial diastólica; PAM = pressão arterial média; FC = frequência cardíaca.

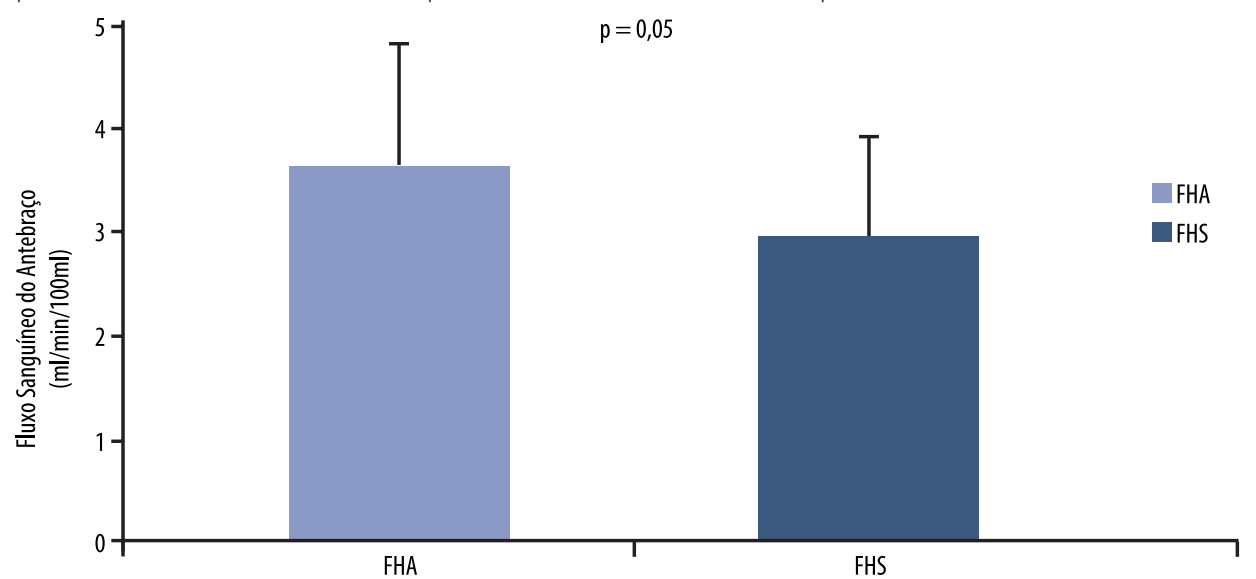

FIGURA 1 - Fluxo sanguíneo do antebraço dos sujeitos fisicamente ativos (FHA) e sedentários (FHS).

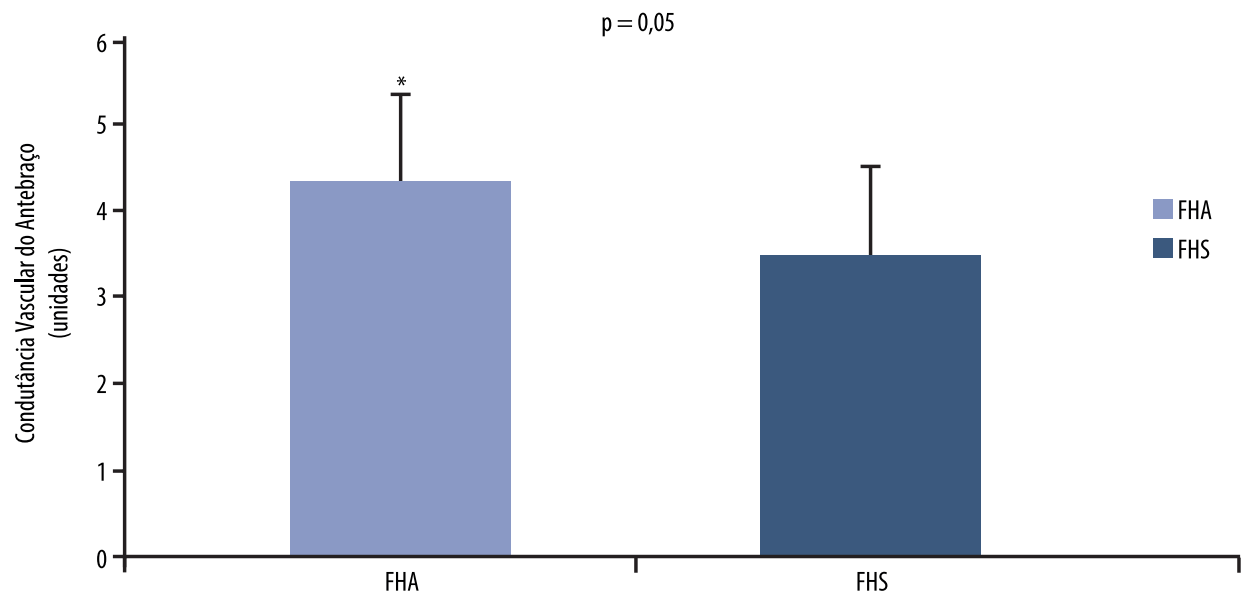

FIGURA 2 - Condutância vascular do antebraço dos sujeitos fisicamente ativos (FHA) e sedentários (FHS). * = diferença significativa em relação ao grupo FHS $(p=0,05)$. 
TABELA 2 - Tamanho do efeito das variáveis de função vascular.

\begin{tabular}{lcc}
\hline Variáveis & Tamanho do efeito & Classificação \\
\hline Fluxo Sanguíneo do Antebraço $(\mathrm{ml} / \mathrm{min} / 100 \mathrm{ml})$ & 0,67 & Elevado \\
\hline Condutância Vascular do Antebraço (unidades) & 0,75 & Elevado \\
\hline
\end{tabular}

\section{Discussão}

Tem sido descrito na literatura que indivíduos com histórico familiar positivo para hipertensão arterial apresentam algumas alterações negativas em mecanismos de controle hemodinâmico ${ }^{5-11}$. Embora muito discutido na hipertensão $0^{19,20}$, pouco foi estudado sobre os benefícios que a prática regular de exercício físico pode promover para a população geneticamente predisposta a desenvolver a doença. Assim, esse foi o primeiro estudo a avaliar a condutância vascular do antebraço em indivíduos fisicamente ativos filhos de hipertensos. O principal achado do presente estudo é que indivíduos fisicamente ativos filhos de hipertensos apresentam maior condutância vascular do antebraço e, consequentemente, melhor função vascular, quando comparados a seus pares sedentários.

Nossos achados podem ser explicados por algumas adaptações benéficas nos vasos sanguíneos, conferidas pela prática regular de exercício físico. Realmente, já é bem estabelecido na literatura que o treinamento físico aeróbio e/ou resistido é capaz de promover melhora estrutural e/ou funcional nos vasos arteriais de indivíduos saudáveis ${ }^{12,16}$ e com doenças cardiovasculares ${ }^{13,15,19,20}$. Nesse contexto, estudos que aplicaram treinamento físico em população saudável, porém filhos de um ou ambos os pais hipertensos, observaram melhoras em alguns mecanismos de controle da função vascular que podem justificar nossos resultados ${ }^{21,22}$. Estudo conduzido por Ciolac et al. ${ }^{21}$ observou o efeito do treinamento físico aeróbio realizado em esteira ergométrica sobre os níveis de óxido nítrico, endotelina-1, noradrenalina e velocidade de onda de pulso em mulheres jovens, filhas de hipertensos. Nessa pesquisa, foi aplicado treinamento físico intervalado de alta intensidade, prescrito por intervalos alternados de 2 minutos com intensidade no limiar anaeróbio (50-60\% do $\mathrm{VO}_{2}$ máximo) e 1 minuto com intensidade no ponto de compensação respiratória (80-90\% do $\mathrm{VO}_{2}$ máximo), durante 40 minutos, e treinamento físico contínuo de moderada intensidade, prescrito entre $60-70 \%$ do $\mathrm{VO}_{2}$ máximo, durante o mesmo período de tempo. Os autores concluíram que o treinamento físico intervalado de alta intensidade e contínuo de moderada intensidade, realizados durante 16 semanas, reduziram significativamente os valores de repouso de noradrenalina e endotelina-1, mas somente o treinamento físico intervalado de alta intensidade foi capaz de aumentar os níveis de óxido nítrico. De maneira semelhante, somente o treinamento físico intervalado de alta intensidade diminuiu significativamente a velocidade de onda de pulso, método padrão ouro para avaliar a rigidez arterial, a ponto de igualar aos valores do grupo controle composto por filhas de normotensos que permaneceram sedentárias durante o mesmo período de pesquisa.

Similarmente, Goldberg et al. ${ }^{22}$ observaram em homens jovens filhos de hipertensos o efeito do treinamento físico aeróbio realizado em cicloergômetro de membros inferiores e prescrito a $65 \%$ da frequência cardíaca máxima, com sessões de 30 minutos, três vezes por semana, durante quatro semanas. Os resultados encontrados corroboram mais uma vez os nossos achados. Os autores concluíram que apenas quatro semanas de treinamento físico foram suficientes para promover alterações benéficas na estrutura dos vasos, com melhora da rigidez arterial e em sua função, com melhora da função endotelial, nesse caso avaliada pela técnica 
de hiperemia reativa. Nesse mesmo estudo, apesar de todos os benefícios verificados, não foi possível observar aumento do fluxo sanguíneo do antebraço com o treinamento físico proposto, concordando com os nossos resultados. Porém, de maneira diferente do estudo de Goldberg et al. ${ }^{22}$, nós corrigimos o valor do fluxo sanguíneo do antebraço pela pressão arterial média, a fim de retirar a influência do nível pressórico sobre a vasodilatação observada.

Além disso, Lenárd et al. ${ }^{7}$ avaliaram, transversalmente, indivíduos sedentários filhos de hipertensos e treinados em diversas modalidades, de maneira semelhante ao presente estudo. Os autores concluíram que os filhos de hipertensos treinados apresentam menor velocidade de onda de pulso e maior coeficiente de distensibilidade, medidas de rigidez arterial, comparados a seus pares sedentários. E mais, os autores observaram que a melhora na estrutura do vaso arterial nos filhos de hipertensos treinados estava positivamente associada com a melhora da sensibilidade barorreflexa, medida de maneira espontânea. Dessa forma, é plausível pensarmos que a inibição tônica que o controle barorreflexo da pressão arterial exerce sobre a atividade nervosa simpática estará íntegra em indivíduos com histórico familiar positivo para hipertensão arterial, quando treinados, culminando em redução da ação vasoconstritora do sistema nervoso simpático. Ainda, levando-se em consideração o achado de Lenárd et al. ${ }^{7}$, que mostra maior sensibilidade barorreflexa nos filhos de hipertensos treinados, podemos inferir que esses indivíduos apresentarão melhor modulação autonômica cardíaca, verificada por maior atividade parassimpática e reduzida atividade simpática, o que pode explicar os valores reduzidos de frequência cardíaca observados no presente estudo.

O método utilizado para avaliação do fluxo sanguíneo do antebraço, a pletismografia de oclusão venosa, é considerada padrão ouro para avaliar a fisiologia vascular humana ${ }^{23}$, o que valoriza os nossos resultados encontrados. Dessa forma, consideramos o achado de melhora da condutância vascular do antebraço especialmente importante, haja visto o valor preditor dessa medida para o desenvolvimento de hipertensão, bem como seu relevante valor prognóstico ${ }^{24,25}$. Portanto, considerando que indivíduos filhos de hipertensos apresentam de 30 a $50 \%$ de chances de desenvolver hipertensão arterial no futuro ${ }^{4}$, podemos concluir que os benefícios promovidos pelo exercício físico podem diminuir esse risco relativo. Além disso, mostramos que os benefícios encontrados de melhora da função vascular parecem estar associados aos escores de nível de atividade física habitual e à quantidade de horas semanais de prática regular de exercício físico em indivíduos geneticamente predispostos a desenvolver hipertensão arterial.

Uma das possíveis limitações do presente estudo é a não realização da medida direta da capacidade cardiorrespiratória e a heterogeneidade nas modalidades esportivas praticadas pelos indivíduos fisicamente ativos. No entanto, utilizamos o questionário de Baecke ${ }^{22}$, que é um instrumento seguro para fornecer informações em relação ao nível de atividade física ocupacional e de lazer, além de exercício físico sistematizado. Esse questionário já foi validado e seus escores apresentam forte correlação com aptidão cardiorrespiratória. Além disso, a ausência da avaliação clínica direta dos pais dos voluntários pode ser considerada uma limitação do nosso estudo. No entanto, o diagnóstico clínico de hipertensão arterial, bem como o uso de medicamentos anti-hipertensivos pelos pais dos voluntários foi confirmado por meio de anamnese, mesmo método de controle de outros estudos já publicados na literatura ${ }^{5,7}$.

Outro fator que poderia interferir nos nossos resultados é a proporção dos sexos entre os grupos. Em nosso estudo, apesar de os grupos serem semelhantes, 
estatisticamente, quanto ao sexo, é possível observar que o grupo de indivíduos fisicamente ativos filhos de hipertensos apresenta maior número de indivíduos do sexo masculino, enquanto no grupo sedentário ocorre o inverso. No entanto, esse fato valoriza ainda mais os resultados sobre a condutância vascular do antebraço uma vez que as mulheres, comparadas aos homens, respondem com maior vasodilatação da artéria braquial mediada por diferentes mecanismos, como ativação de receptores $\beta 2$-adrenérgicos, em resposta à acetilcolina e à hiperemia reativa ${ }^{26,27}$. Assim, o maior número de mulheres no grupo FHS poderia prejudicar as diferenças entre os grupos investigados, o que não aconteceu.

Com os resultados observados, pode-se concluir que indivíduos fisicamente ativos filhos de hipertensos apresentam melhor função vascular, medida em condições de repouso, quando comparados a indivíduos sedentários filhos de hipertensos.

\section{Contribuição dos autores}

Os autores declaram que participaram ativamente do planejamento, coleta dos dados e escrita do artigo. Leonardo Barbosa de Almeida participou da análise e interpretação dos dados, redação do artigo e aprovação da versão final a ser publicada. Isabelle Magalhães Guedes Freitas, Livia Victorino de Souza, Pedro Augusto de Carvalho Mira, Daniel Godoy Martinez e Mateus Camaroti Laterza participaram da concepção do projeto, análise e interpretação dos dados, revisão crítica relevante do conteúdo intelectual e aprovação da versão final a ser publicada.”

\section{Referências}

1. Wang NY, Young JH, Meoni LA, Ford DE, Erlinger TP, Klag MJ. Blood pressure change and risk of hypertension associated with parental hypertension. Arch Intern Med. 2008; 168: 643-48.

2. Chobanian AV, Bakris GL, Black HR, William C, Cushman LA, Jackson TW et al. Seventh report of the Joint National Committee on Prevention, Detection, Evaluation, and Treatment of High Blood Pressure. Hypertension. 2003; 42: 1206-52.

3. Timberlake DS, O'Connor, DT, Parmer RJ. Molecular genetics of essential hypertension: recent results and emerging strategies. Curr Opin Nephrol Hypertens. 2001: 10: 71-9.

4. Mozaffarian D. Heart disease and stroke statistics - 2015 Update: A report from the American Heart Association. Circulation. 2015; 131: e29-e322.

5. Pitzalis MV, Iacoviello M, Massari F, Guida P, Romito R, Forleo C et al. Influence of gender and family history of hypertension on autonomic control of heart rate, diastolic function and brain natriuretic peptide. J Hypertens. 2001; 19: 143-8.

6. Lopes HF, Bortolotto LA, Szlejf C, Kamitsuji CS, Krieger EM. Hemodynamic and metabolic profile in offspring of malignant hypertensive parents. Hypertension. 2001; 38: 616-20.

7. Lenard Z, Studinger P, Mersich B, Kollai M. Cardiovagal autonomic function in sedentary and trained offspring of hypertensive parents. J Physiol. 2005; 565: 1031-8.

8. Ciolac EG, Bocchi EA, Bortolotto LA, Carvalho VO, Greve JMD, Guimarães GV. Haemodynamic, metabolic and neuro-humoral abnormalities in young normotensive women at high familial risk for hypertension. J Hum Hypert. 2010; 24: 814-22.

9. Evrengul H, Tanriverdi H, Kilic ID, Dursunoglu D, Ozcan EE, Kaftan A et al. Aortic stiffness and flow-mediated dilatation in normotensive offspring of parents with hypertension. Cardiol Young. 2012; 22: 451-56.

10. Kyvelou SMG, Vyssoulis GP, Karpanou EA, Adamopoulos DN, Cokkinos V, Stefanadis CI. Arterial stiffness in offspring of hypertensive parents: A pilot study. Int J Cardiol. 2008; 129: 438-40.

11. Bernatova I, Csizmadiova Z, Kopincova J, Puzserova A. Vascular function and nitric oxide production in chronic social-stress-exposed rats with various family history of hypertension. J Physiol Pharmac. 2007; 58: 487-501. 
12. Dinenno FA, Tanaka H, Monahan KD, Clevenger CM, Eskurza I, DeSouza CA et al. Regular endurance exercise induces expansive arterial remodelling in the trained limbs of healthy men. J Physiol. 2001; 534: 287-95.

13. Naoki F, Prasad A, Hastings JL, Bhella PS, Shibata S, Palmer D et al. Cardiovascular effects of 1 year of progressive endurance exercise training in patients with heart failure with preserved efection fraction. Am Heart J. 2012; 146: 869-77.

14. Sartori TE, Nunes RAB, Silva GT, Silva SC, Rondon MUPB, Negrão CE et al. Influence of demographic and metabolic variables on forearm blood flow and vascular conductance in individuals without overt heart diseases. Vasc Health Risk Manag. 2010; 6:431-7.

15. Hambrecht R, Fiehn E, Weigl C, Gielen S, Hamann C, Kaiser R et al. Regular physical exercise corrects endothelial dysfunction and improves exercise capacity in patients with chronic heart failure. Circulation. 1998; 98: 2709-15.

16. Galetta F, Franzoni F, Virdis A, Ghiadoni L, Taddei S, Salvetti A et al. Endotheliumdependent vasodilatation and carotid artery wall remodeling in athletes and sedentary subjects. Atherosclerosis. 2006; 196: 184-92.

17. Florindo AA, Latorre MRDO. Validation and reliability of the Baecke questionnaire for the evaluation of habitual physical activity in adult men. Rev Bras Med Esporte. 2003; 9: 129-35.

18. Ghetti FF, Lacerda RP, Werneck FZ, Coelho EF, Vaisman M, Lima JRP et al. Impairment of muscle vasodilation during mental stress in women with subclinical hypothyroidism. Arq Bras Endocrinol Metab. 2014; 58: 750-57.

19. Laterza MC, Matos LDNJ, Trombetta IC, Braga AMW, Roveda F, Alves MJNN et al. Exercise training restores baroreflex sensitivity in never-treated hypertensive patients. Hypertension. 2007; 49: 1298-306.

20. Cornelissen VA, Buys R, Smart NA. Endurance exercise beneficially affects ambulatory blood pressure: A systematic review and meta-analysis. J Hypertens. 2013; 31: 639-48.

21. Ciolac EG, Bocchi EA, Bortolotto LA, Carvalho VO, Greve JMD, Guimarães GV. Effects of high-intensity aerobic interval training vs. moderate exercise on haemodynamic, metabolic and neuro-humoral abnormalities of young normotensive women at high familial risk for hypertension. Hypert Res. 2010; 33: 836-43.

22. Goldberg MJ, Boucther SH, Boutcher YN. The effect of 4 weeks of aerobic exercise on vascular and baroreflex function of young men with family history of hypertension. $\mathrm{J}$ Hum Hypert. 2012; 26: 644-49.

23. Wilkinson IB, Webb DJ. Venous occlusion plethysmography in cardiovascular research: methodology and clinical applications. J Clin Pharmacol. 2001; 52: 631-46.

24. Forte P, Benjamin N. Does an impaired flow mediated vasodilatation predict hypertension in offspring hypertensive parents? Heart. 2001; 85: 131-32.

25. Fagard RH, Pardaens K, Staessen JA, Thijs L. Prognostic Value of Invasive Hemodynamic Measurements at Rest and During Exercise in Hypertensive Men. Hypertension.1996; 28: 31-36.

26. Kneale BJ, Chowienczyk PJ, Brett SE, Coltart DJ, Ritter JM. Gender differences in sensitivity to adrenergic agonists of forearm resistance vasculature. J Amer Coll Cardiol. 2000; 36: 1233-38.

27. Parker BA, Smithmyer SL, Perlberg JA, Mishkin AD, Herr MD, Proctor DN. Sex differences in leg during graded knee extensor exercise in young adults. J Appl Physiol. 2007; 103: 1583-91.

ENDEREÇO PARA CORRESPONDÊNCIA MATEUS CAMAROTI LATERZA mateuslaterza@hotmail.com
Faculdade de Educação Física e

Desportos, Universidade Federal de

Juiz de Fora.

Campus Universitário, Martelos

Juiz de Fora, MG, Brasil.

Telefone: (32) 2102-3291 